\title{
Dichlorodiphenyltrichloroethane Specifically Depletes Dopaminergic Neurons in Primary Cell Culture
}

\author{
K.W. Leung a Y.S. Chan ${ }^{b}$ K.K.L. Yung ${ }^{a}$ \\ a Department of Biology and Institute for Natural Resources and Environmental Management, \\ Hong Kong Baptist University, Kowloon Tong, and ' ${ }^{2}$ Department of Physiology, Faculty of Medicine, \\ The University of Hong Kong, Hong Kong, China
}

\section{Key Words}

Dichlorodiphenyltrichloroethane • Neurotoxicity •

Pesticide $\cdot$ Insecticide $\cdot$ Parkinson's disease $\cdot$ Neuronal cell death

\begin{abstract}
Toxicity of dichlorodiphenyltrichloroethane (DDT) to dopaminergic neurons in primary cell culture was investigated in the present study. Developing neurons from the substantia nigra of neonatal rats were cultured. After treatments with different concentrations of DDT (5$12.5 \mu M)$, specific cell death of tyrosine-hydroxylaseimmunoreactive dopaminergic neurons was observed in the culture by flow cytometric analysis. More than $60 \%$ of dopaminergic neurons were depleted after treatments with 10 and $12.5 \mu \mathrm{M}$ of DDT. In addition, significant reductions of intensity levels of tyrosine hydroxylase immunofluorescence were observed in dopaminergic neurons after DDT treatments even at low concentrations of DDT. The present findings indicate that dopaminergic neurons are more susceptible to DDT toxicity than other types of neurons in the primary cell culture. Moreover, it is shown that the synthesis of dopamine in dopa-
\end{abstract}

\section{KARGER}

Fax + 41613061234

E-Mail karger@karger.ch

www.karger.com

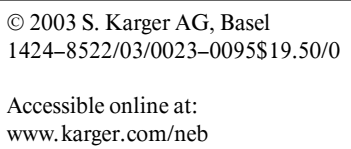

minergic neurons is also depressed. Previous studies have demonstrated that perinatal exposure of DDT causes neurons to be more susceptible to neurotoxic damages in later adult life. The present findings thus provide evidence that dopaminergic neurons that are undergoing growth and development are targets of DDT neurotoxic effects. Exposure to DDT from contaminated environments is therefore a potential risk of onset of Parkinson's disease.

Copyright ( 2003 S. Karger AG, Basel

\section{Introduction}

$\mathrm{p}, \mathrm{p}^{\prime}$-Dichlorodiphenyltrichloroethane (DDT) was a widely used pesticide and it has been banned in many developed countries since 1972 due to potential hazards to human health and the environment [1]. However, DDT is still produced and exported by some developing countries for the control of infectious diseases and other public health purposes [2]. A growing body of evidence has indicated that DDT and its metabolites are potent carcinogens and endocrine disruptors that can cause serious health problems in humans [1,2]. DDT is a persistent 
compound and is known to bio-accumulate in adipose tissues in animals [1, 2]. Different levels of accumulation in human tissues are found among human populations in the world and may still present a potential threat to human health [1].

Parkinson's disease is a common human motor disorder that is caused by a specific degeneration of dopaminergic neurons in the substantia nigra of the basal ganglia [3]. A number of potential causes of Parkinson's disease including genetic, chemical and environmental factors have been suggested [4-9]. The etiology of Parkinson's disease, however, remains largely unknown $[4,5]$. Recent evidence indicates that exposure to environmental toxins such as pesticides may cause Parkinson's disease [6-8].

Previous studies have already indicated that DDT has potent neurological effects and that exposure to DDT can cause onset of parkinsonian-like symptoms [10-13]. DDT is known to induce tremor in animals $[10,11]$ and cause depletion in dopamine levels in the brain [12, 13]. Depletion of dopamine levels in the striatum in rats after DDT exposure is found to be similar to the effects of 1-methyl4-phenyl-1,2,3,6-tetrahydropyridine, a well-known neurotoxin that can cause degeneration of dopaminergic neurons [14]. In addition, perinatal exposure to DDT has been found to result in an increase in susceptibility to damage of neurotoxins in adulthood [15]. This is important as exposure to DDT and other related toxins often occurs through breast milk feeding [1]. Moreover, early insults on neurons during embryonic and perinatal development are known to cause neuronal damages in later stages of life [16, 17].

Despite these previous observations, the detailed neurotoxicological effects of DDT on dopaminergic neurons and whether exposure to DDT can induce a specific cell death of dopaminergic neurons in the brain remain unknown. The objectives of the present study were to investigate the effects of DDT exposure to neurons, in particular the dopaminergic neurons, in primary cell culture. Tyrosine hydroxylase (TH), a synthetic enzyme for dopamine, is a well-established marker for nigral dopaminergic neurons [18]. In the present study, immunoreactivity for $\mathrm{TH}$ was employed as a specific marker for nigral dopaminergic neurons. The percentage of cell death of the nigral cell culture as a whole was studied by cytotoxicity assay. The percentage of cell death of TH-positive dopaminergic neurons after DDT treatments was quantified by flow cytometry. In addition, levels of intensity of $\mathrm{TH}$ immunofluorescence in neurons treated with DDT were measured by image analysis.

\section{Materials and Methods}

Primary Cell Culture of Dopaminergic Neurons

In the present study, neonatal rats (postnatal day 1, SpragueDawley) were used. The handling of rats and all procedures that were performed on them were approved, in accordance with the Animals (Control of Experiments) Ordinance, Hong Kong, China, by the Committee on the Use of Human and Animal Subjects in Teaching and Research, Hong Kong Baptist University, and conformed to The Principles of Laboratory Animal Care [NIH publication No. 86-23, revised 1985]. The rats were sacrificed by decapitation and the brain region of the substantia nigra was dissected. Primary neuronal cultures were prepared in accordance with a previous study [19] with slight modifications. Briefly, the brain tissues were chopped using a razor blade in minimal essential medium (Gibco, BRL). The chopped tissues were then incubated in trypsin/EDTA solution with $1 \%$ penicillin-streptomycin (Gibco) and B27 supplement (Gibco) for $1 \mathrm{~h}$ at $37^{\circ} \mathrm{C}$. Agitation of the medium was performed every $15 \mathrm{~min}$. Single cell suspension was obtained by dissociation of the tissues in neurobasal medium with soybean trypsin inhibitor (Gibco) and DNase 1 (Boehringer Mannheim) for $30 \mathrm{~min}$ at $37^{\circ} \mathrm{C}$. Cell pellets were obtained by centrifugation (1,200 rpm for $5 \mathrm{~min})$. The pellets were washed and resuspended in culture medium (neurobasal medium, $1 \%$ penicillin-streptomycin, B27 supplement; Gibco, and 10\% fetal bovine serum; Sigma). The neurons were then plated on poly- $D$ lysine-coated 6-well plastic plates or on glass coverslips. The cultures were maintained at $37^{\circ} \mathrm{C}$ in $\mathrm{CO}_{2}$ incubator $\left(5 \% \mathrm{CO}_{2}\right)$. Cytosine arabinoside $(10 \mu M$, Fluka) was added to the culture 3 days after plating in order to inhibit the growth of glial cells [20]. DDT treatments on the cultures were conducted at 6 days in vitro (DIV).

DDT Treatments

Nigral neurons in culture at 6 DIV were treated with p,p'-DDT (ChemService Inc.) of different concentrations $(0.1-12.5 \mu M)$ in culture medium. The neurons were incubated in DDT solutions for $24 \mathrm{~h}$ at $37^{\circ} \mathrm{C}$ in $\mathrm{CO}_{2}$ incubator $\left(5 \% \mathrm{CO}_{2}\right)$ prior to the following procedures.

\section{Lethal Concentration 50 of DDT for Nigral Neurons}

The lethal concentration 50 (LC50) of DDT for cultured nigral neurons was determined by the enzymatic activity of lactate dehydrogenase (LDH) in the culture medium using a commercially available cytotoxicity detection kit (cytotoxicity detection kit LDH, Roche Molecular Biochemicals). Protocols were employed in accordance with the supplier's information. The percentage of cell death was estimated by measuring the optical density of the tested samples using a spectrophotometer (iEMS analyzer, Labsystems).

Quantification of Dopaminergic Neurons in Nigral Cell Cultures

In nigral cell cultures without DDT treatment, neurons in suspension were fixed with fixative (4\% paraformaldehyde in $0.1 \mathrm{M}$ phosphate buffer, $\mathrm{pH}$ 7.4) for $20 \mathrm{~min}$ at room temperature. The cells were then pelleted by centrifugation at 1,200 rpm for $5 \mathrm{~min}$, washed and resuspended in phosphate buffered saline (PBS) prior to immunofluorescence. Neurons were labeled to show TH immunoreactivity using commercially available antibodies (primary antibody: mouse monoclonal, 1:2,000 in PBS-Triton, Chemicon; secondary antibody: goat-antimouse IgG conjugated with Alexa 488, 1:100 in PBS-Triton, Molecular Probes). Flow cytometric analysis was performed using a FACScan flow cytometer (Becton-Dickinson) [21, 22]. During the 
analysis, 10,000 events were routinely collected. Flow cytometry was calibrated by counting 10,000 events without primary antibody with less than $5 \%$ of cells displaying a high level of fluorescence. In those immunostained cells, the number of TH-immunopositive cells was determined. The percentage of TH-positive dopaminergic neurons was obtained. Data were expressed as mean percentage \pm standard error of the mean (SEM).

\section{Quantification of Cell Death of Dopaminergic Neurons after}

\section{DDT Treatments}

After DDT treatments $(5,10$ and $12.5 \mu M)$, the number of THpositive neurons in the nigral cell culture was also determined by flow cytometric analyses as described above. The percentage of dopaminergic neurons in the culture was then determined. Data were expressed as mean percentage \pm SEM. Statistical analysis of the treatments with different concentrations of DDT was performed by one-way ANOVA with Duncan's multiple range tests.

\section{Determination of Intensity of TH Immunofluorescence after} DDT Treatments

After DDT treatments, neurons were fixed in fixative (4\% paraformaldehyde in $0.1 \mathrm{M}$ phosphate buffer, $\mathrm{pH}$ 7.4) for $20 \mathrm{~min}$ at room temperature. Neurons were washed $(3 \times \mathrm{PBS}, \mathrm{pH}$ 7.4) prior to immunofluorescence. TH immunoreactivity was revealed using the same commercially available antibodies as described previously [23]. Images of TH-positive neurons were captured under the same parameters in a fluorescence microscope. Levels of TH immunofluorescence were determined using an image-analyzing software (Metamorph, Universal Imaging) as described previously [23]. Intensity of TH immunofluorescence was averaged and expressed as means \pm SEM and statistical analyses were performed using one-way ANOVA with Duncan's multiple range tests.

\section{Results}

\section{Development of Nigral Neurons in Primary Cell}

\section{Culture}

The size of the neuronal perikarya in cell culture was found to be increased during incubation in culture medium from 1 to 6 DIV (fig. 1A-F). The number of neurites was also seen to increase during development in cell culture (fig. 1A-F).

\section{Effects of DDT Treatments on Total Nigral Neurons}

The LC50 of DDT for nigral neurons in primary cell culture was determined as $12.8 \mu M$ by LDH assays. The number of neurons was seen to be reduced after different concentrations of DDT treatments (fig. 2A-D). A decrease in nigral neurons was found in cultures treated with $5 \mu M$ of DDT (fig. 2B). Fewer numbers of nigral neurons were seen in cultures with higher concentrations of DDT (fig. 2C-D).

DDT Depletes Cultured Dopaminergic

Neurons

\section{Significant Reduction of TH-Positive Dopaminergic} Neurons after DDT Treatments

Results of the flow cytometric analysis showed that there was differential cell death among the heterogeneous subpopulations of cells in the nigral primary cell culture (fig. 3). In control analyses where TH antibody was omitted, less than $5 \%$ of cells were found to exhibit a high level of fluorescence (fig. 3A). These cells were presumed to display autofluorescence and this was treated as the background. In those cultures immunostained to reveal $\mathrm{TH}$ immunoreactivity, about $36 \%$ of $\mathrm{TH}$-immunoreactive cells were found in the primary nigral culture (fig. 3B, 4A).

After treatment with $5 \mu M$ of DDT, the percentage of TH-positive dopaminergic neurons in the cell culture was reduced to around 29\% (fig. 3C, 4A). However, it was not statistically significant (fig. 4A). After treatments with 10 and $12.5 \mu M$ of DDT, the percentage of TH-positive cells in the primary cell cultures was found to be significantly reduced to $18-16 \%$, respectively (fig. 3D-E, 4A).

Statistical analysis also showed that $5 \mu M$ of DDT induced about $20 \%$ cell death of TH-positive dopaminergic cells in the cell culture (fig. 4B). An increased amount of DDT (10-12.5 $\mathrm{mM})$ induced more than $60 \%$ cell death of TH-positive dopaminergic cells in the cultures (fig. 4B). Significant differences were seen among the treatments in a dose-dependent manner (fig. 4B).

\section{Significant Reduction of TH Immunofluorescence after DDT Treatments}

Image analysis revealed that the intensity levels of $\mathrm{TH}$ immunofluorescence were also reduced after DDT treatments. Significant reduction of the level of fluorescence was found in cells treated with $5 \mu M$ of DDT (fig. 5). Much lower levels of fluorescence were seen in cells treated with 10 and $12 \mu M$ of DDT (fig. 5).

\section{Discussion}

The present results provide the novel and interesting observation that in vitro DDT treatments induce specific cell death of TH-positive dopaminergic neurons in primary cell culture. There is evidence that dopaminergic neurons during growth and development are targets of DDT toxic effects. These findings might also indicate that exposure to DDT may contribute to the onset of Parkinson's disease in humans.

The nigral primary cell culture is a heterogeneous culture. The present results indicate that around $36 \%$ of cells

Neuroembryology 2003;2:95-102 

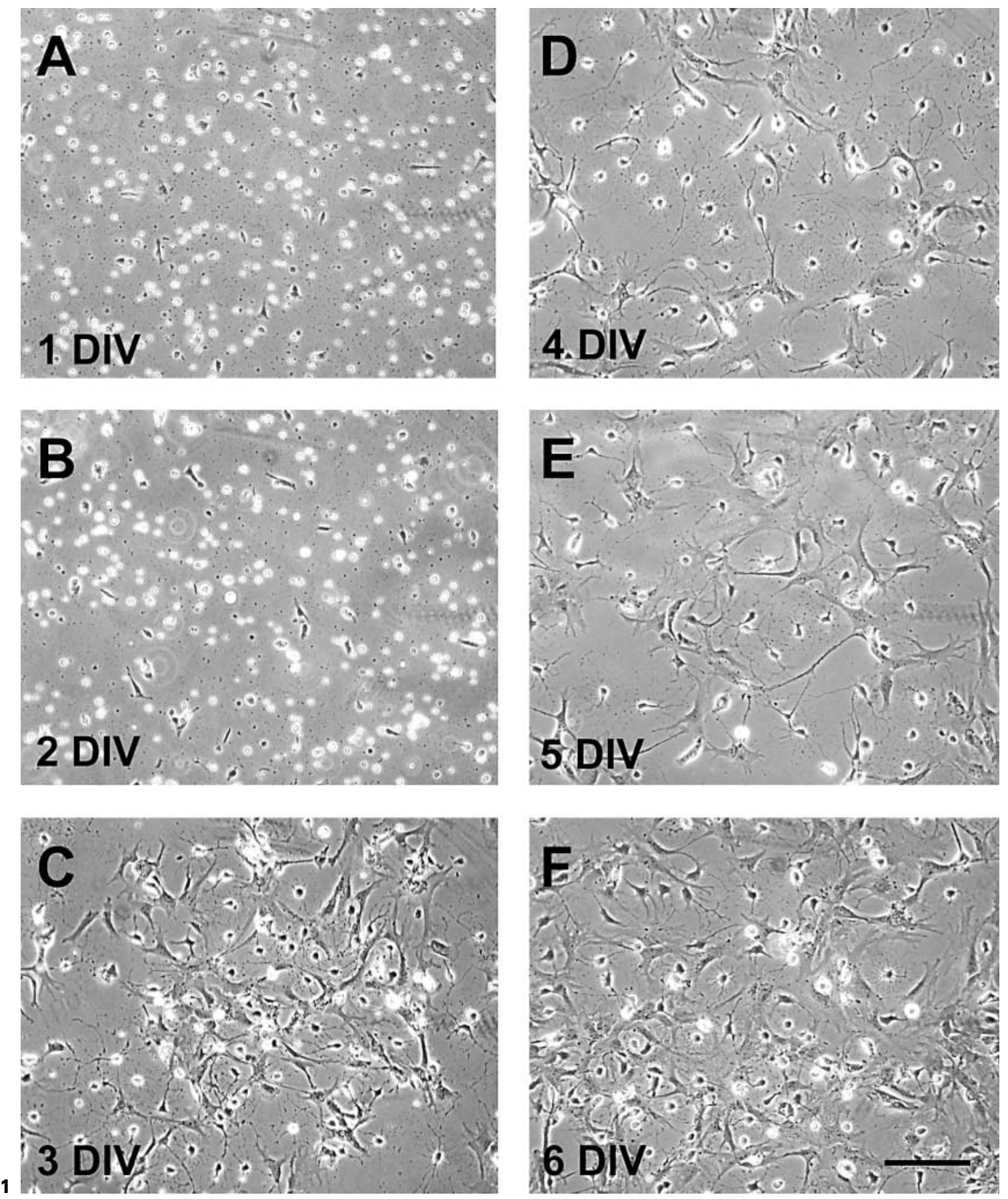

Fig. 1. Growth of neurons in primary cell culture of the rat substantia nigra. Scale bar (valid for all micrographs): $500 \mu \mathrm{m}$.

Fig. 2. Depletion of total nigral neurons after DDT treatment. In control (A), nigral neurons are abundant. A slight reduction of neurons is seen after treatment with $5 \mu M$ DDT (B). Much higher reductions in nigral cell number are seen after treatments with high concentrations of DDT (C-D). Scale bar (valid for all micrographs): $500 \mu \mathrm{m}$.

Fig. 3. Flow cytometric analysis of the changes of TH-immunoreactive dopamine neurons in culture after DDT treatments. In the con- trol experiments with no primary antibody against $\mathrm{TH}$, only a few fluorescent cells are seen (represent by green dots in A). Immunonegative cells are indicated in red. In nigral culture without DDT treatment, a great number of TH-positive neurons are found (B). After treatment with $5 \mu M$ of DDT, reduction of TH-positive neurons is seen $(\mathbf{C})$. After treatments with higher concentrations of DDT (10-12.5 $\mu M)$, only a few TH-positive neurons remained (D, E). SSC-H = Side-scattered value; FL1-H = fluorescence intensity. Arrows indicate the cutoff fluorescence intensity of TH-immunopositive cells. 

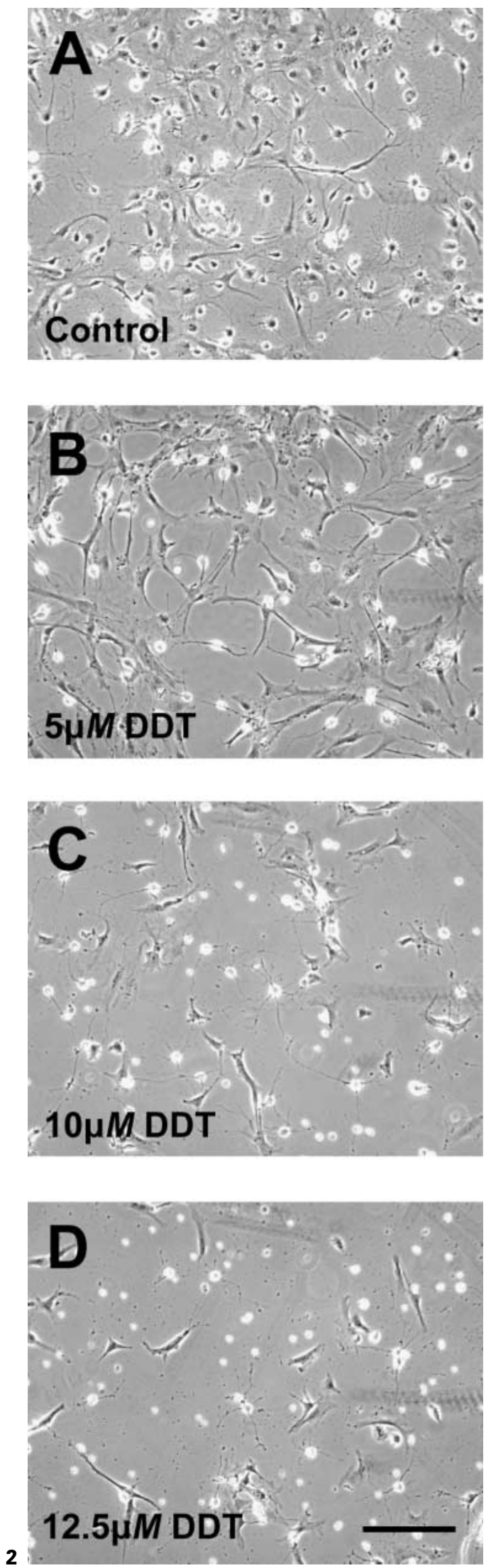

DDT Depletes Cultured Dopaminergic Neurons
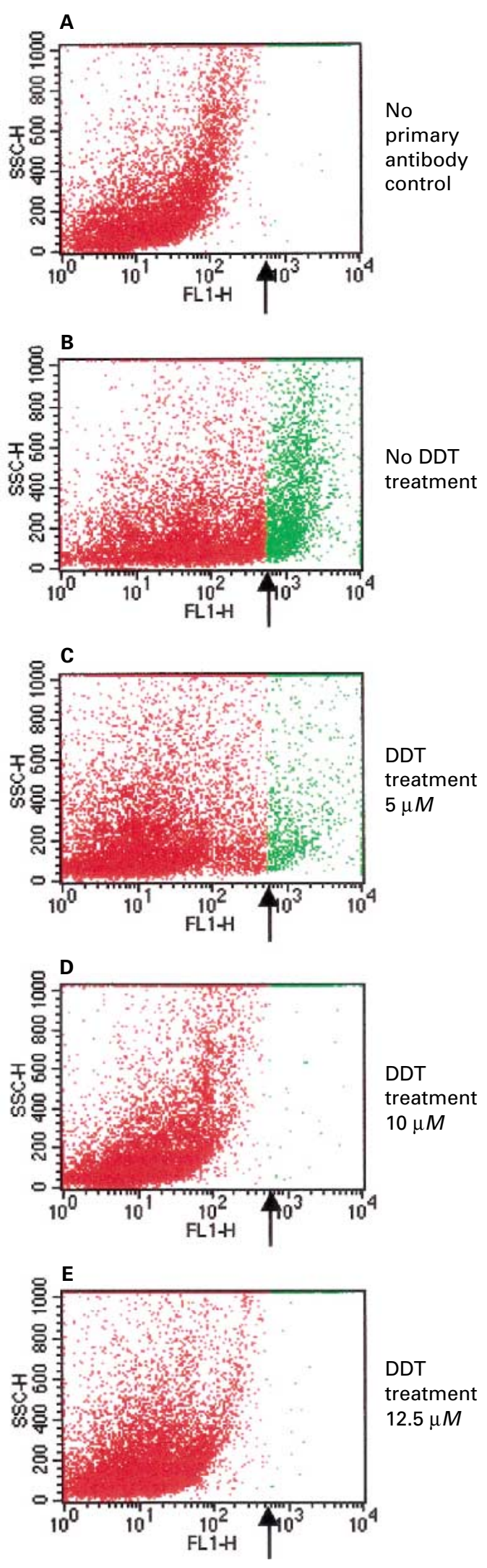

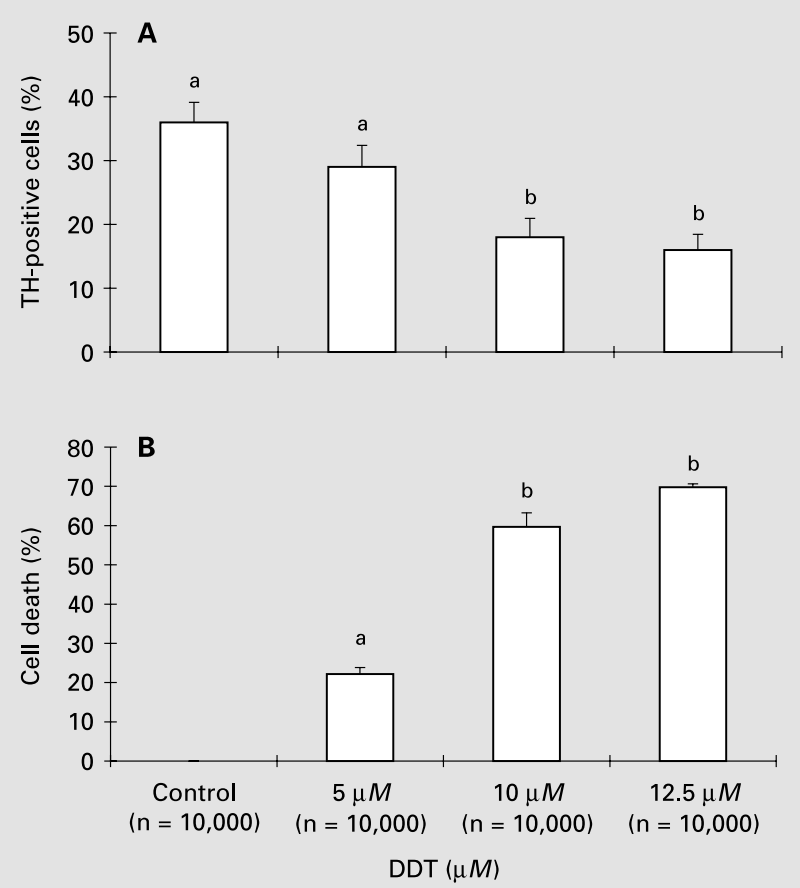

Fig. 4. A Percentage of TH-positive neurons in primary cell culture. Changes in the percentage of TH-positive cells in the cell culture quantified by flow cytometer. B Percentage of depletion of TH-positive neurons in primary cell culture. Changes in the percentage of depletion of TH-positive neurons after DDT treatments. Bars in both graphs represent mean percentage \pm SEM. Statistical analysis is performed using one-way ANOVA with Duncan's multiple range tests. Bars with different letters indicate that a significant difference $(\mathrm{p}<$ 0.05 ) is found between the data. $\mathrm{n}=$ Number of cells counted.

in the culture are found to be TH-positive dopaminergic neurons, which is consistent with a previous study [24].

Previous studies have long indicated that DDT can induce parkinsonian symptoms $[10,11]$ and modulation of dopamine release in the brain [11-13]. The present findings thus extend the previous findings and confirm that exposure to DDT induce specific cell death of dopaminergic neurons. One important point is that even at a low concentration of DDT (around $5 \mu M$ ), there is already a $20 \%$ depletion of dopaminergic neurons. More than $60 \%$ of dopaminergic neurons are depleted at DDT concentrations $(10-12.5 \mu M)$ that approach the LC50 value $(12.8 \mu M)$. In the heterogeneous nigral cell culture as a whole, $12.8 \mu M$ of DDT induces only about $50 \%$ cell death. With respect to the dopaminergic neurons, more than $60 \%$ cell death is observed. This indicates that DDT

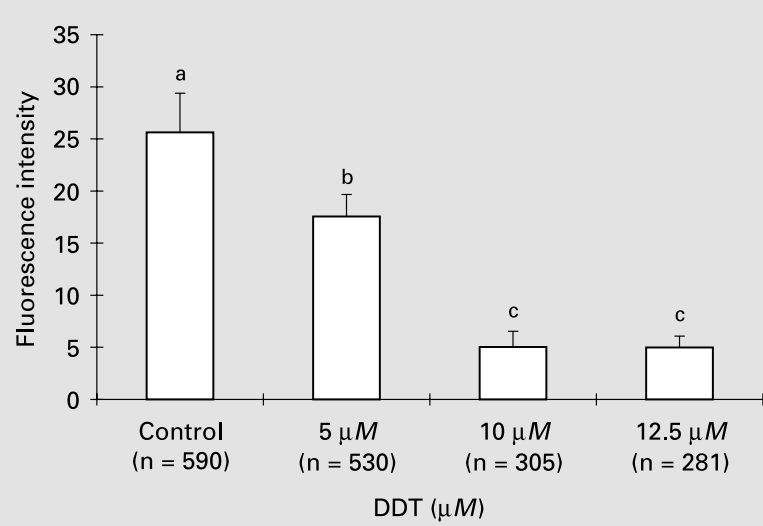

Fig. 5. Depletion of fluorescence intensity of TH-positive neurons. Changes in the levels of intensity of TH immunofluorescence after DDT treatments. Bars represent mean fluorescence intensity \pm SEM. Bars with different letters indicate that a significant difference $(p<0.05)$ is found between the data by one-way ANOVA with Duncan's multiple range tests. $\mathrm{n}=$ Number of cells measured.

has specific effects on dopaminergic neurons versus other neurons or related cells in the mixed primary cell culture. Parkinsonian patients will show clinical symptoms when $50 \%$ of dopaminergic neurons have degenerated in their substantia nigra [4]. It is worth noting that there is a considerable difference between the in vivo and in vitro conditions. However, the present findings thus imply that there is a close relationship between exposure to DDT and onset of Parkinson's disease.

Evidence has already indicated that an early exposure to toxins during embryonic development can be one of the factors that results in neuronal degeneration in later adult stages $[16,17]$. Motor deficits are among those neurological diseases that are caused by early exposures to neurotoxins [16]. DDT is found to be one of those neurotoxins that are potentially harmful to neurons during growth and development $[15,25,26]$. Exposure of fetal and neonatal neurons to DDT and other neurotoxins may act through maternal routes, i.e., either by placental or milk feeding $[1,16,17]$. Our present findings thus imply that an early exposure to DDT may relate to the onset of Parkinson's disease in later stages of life.

Previous studies have found that the target sites of DDT in neurons are sodium channels in the cell membrane of axons [27-29]. The major actions of DDT are prolongation of the opening of the sodium channels that leads to overexcitation of neurons [27-29], subsequently 
resulting in cell death of neurons. The present findings indicate that DDT at around $12.8 \mu M$ is able to cause a significant level of cell death of nigral neurons. The cause of cell death is likely due to the overexcitation mechanism mentioned above.

Interestingly, previous studies have also found that DDT depresses the phosphorylation efficiency of mitochondria in nonneuronal cells, which consequently leads to depression of mitochondrial respiratory functions [30, 31]. Recently, investigations have provided a crucial correlation between mitochondrial dysfunctions in dopaminergic neurons that is caused by exposure to insecticides and the onset of Parkinson's disease [5-7]. These lines of evidence match our present findings which indicate that DDT differentially induces a higher percentage of cell death in dopaminergic neurons. Our results thus provide evidence that dopaminergic neurons are more susceptible to DDT toxicity than other types of neurons. Importantly, our preliminary observations of DDT toxicity in other types of neurons, namely neurons from the striatum, indicate that striatal neurons in primary culture are less vulnerable to DDT toxicity than dopaminergic neurons [Leung and Yung, unpubl. results]. Exposure to DDT from a contaminated environment is therefore a realistic and potential risk for the onset of Parkinson's disease.

In addition, our findings also indicate that DDT induces a significant decrease in $\mathrm{TH}$ immunofluorescence in surviving dopaminergic neurons. Significant depletion of TH immunoreactivity occurs at a relatively low concentration of DDT (around $5 \mu M$ ). As discussed above, this low concentration of DDT does not cause significant cell death in dopaminergic neurons. Depletion of TH immunoreactivity implies that there may be a reduction in dopamine synthesis in dopaminergic neurons during growth and development. Thus, DDT may cause a reduction in dopamine synthesis prior to toxic cell death of the dopaminergic neurons. These findings indicate that DDT can also affect the dopamine metabolism in dopaminergic neurons even at lower concentrations. The present findings therefore extend the previous observations that DDT depresses dopamine release [11-13]. However, the precise mechanisms of how DDT interacts with dopamine synthesis remain to be established.

In conclusion, the present findings demonstrate that DDT specifically depletes dopaminergic neurons in primary cell culture. This could lead to the onset of Parkinson's disease. Exposure to DDT from the environment, especially in early stages of neuronal growth and differentiation, is a potential risk factor for Parkinson's disease.

\section{Acknowledgements}

The present work was supported by HKBU2/00C central allocation, Research Grant Council, Hong Kong. The authors would like to thank Miss L.Y. Man for technical assistance.

\section{References}

1 Jaga K, Dharmani C: Global surveillance of DDT and DDE levels in human tissues. Int $\mathrm{J}$ Occup Med Environ Health 2003; 16:7-20.

2 Turusov V, Rakitsky V, Tomatis L: Dichlorodiphenyltrichloroethane (DDT): Ubiquity, persistence, and risks. Environ Health Perspect 2002; 110:125-128.

3 Albin RL, Young AB, Penney JB: The functional anatomy of disorders of the basal ganglia. Trends Neurosci 1995;18:63-64.

4 Sherer TB, Betarbet R, Greenamyre JT: Pathogenesis of Parkinson's disease. Curr Opin Investig Drugs 2001;2:657-662.

5 Schapira AH: Causes of neuronal death in Parkinson's disease. Adv Neurol 2001;86:155162.

6 Jenner P: Parkinson's disease, pesticides and mitochondrial dysfunction. Trends Neurosci 2001;24:245-247

7 Greenamyre JT, MacKenzie G, Peng TI, Stephans SE: Mitochondrial dysfunction in Parkinson's disease. Biochem Soc Symp 1999;66: 85-97.
8 Zhang Y, Dawson VL, Dawson TM: Oxidative stress and genetics in the pathogenesis of Parkinson's disease. Neurobiol Dis 2000;7:240250 .

9 Warner TT, Schapira AH: Genetic and environmental factors in the cause of Parkinson's disease. Ann Neurol 2003;53(Suppl 3):S16S23.

10 Herr DW, Tilson HA: Modulation of p,p'DDT-induced tremor by catecholaminergic agents. Toxicol Appl Pharmacol 1987;91:149_ 158.

11 Hong JS, Herr DW, Hudson PM, Tilson HA: Neurochemical effects of DDT in rat brain in vivo. Arch Toxicol Suppl 1986;9:14-26.

12 Hrdina PD, Singhal RL, Peters DA, Ling GM: Role of brain acetylcholine and dopamine in acute neurotoxic effects of DDT. Eur J Pharmacol 1971;15:379-382.

13 Hrdina PD, Singhal RL, Peters DA, Ling GM: Some neurochemical alterations during acute DDT poisoning. Toxicol Appl Pharmacol 1973;25:276-288.
14 Fredriksson A, Fredriksson M, Eriksson P. Neonatal exposure to paraquat or MPTP induces permanent changes in striatum dopamine and behavior in adult mice. Toxicol Appl Pharmacol 1993;122:258-264.

15 Eriksson P, Talts U: Neonatal exposure to neurotoxic pesticides increases adult susceptibility: A review of current findings. Neurotoxicology 2000;21:37-47.

16 Ulfig N: The functional organization of the developing human brains in relation to motor deficits, cognitive impairment and psychotic states. Neuroembryology 2003;2:81-93.

17 Schmitz C, Frank HG: Discrete placental dysfunction: The overlooked player in prenatal roots of neuropsychiatric diseases? Neuroembryology 2002;1:169-175.

18 Freund TF, Powell JF, Smith AD: Tyrosine hydroxylase-immunoreactive boutons in synaptic contact with identified striatonigral neurons, with particular reference to dendritic spines. Neuroscience 1984;13:1189-1215. 
19 Brewer GJ: Serum-free B27/neurobasal medium supports differentiated growth of neurons from the striatum, substantia nigra, septum, cerebral cortex, cerebellum, and dentate gyrus. J Neurosci Res 1995;42:674-683.

20 Lafon-Cazal M, Viennois G, Kuhn R, Malitschek B, Pin JP, Shigemoto R, Bockaert J mGluR7-like receptor and GABA(B) receptor activation enhance neurotoxic effects of $\mathrm{N}$ methyl- $D$-aspartate in cultured mouse striatal GABAergic neurones. Neuropharmacology 1999;38:1631-1640.

21 Drach J, Gattringer C, Glassl H, Schwarting R, Stein H, Huber H: Simultaneous flow cytometric analysis of surface markers and nuclear Ki67 antigen in leukemia and lymphoma. Cytometry $1989 ; 10: 743-749$.
22 Landberg G, Tan EM, Roos G: Flow cytometric multiparameter analysis of proliferating cell nuclear antigen/cyclin and Ki-67 antigen: A new view of the cell cycle. Exp Cell Res 1990; 187:111-118.

23 Lau WK, Yeung CW, Lui PW, Cheung LH, Poon NT, Yung KKL: Different trends in modulation of NMDAR1 and NMDAR2B gene expression in cultured cortical and hippocampal neurons after lead exposure. Brain Res 2002; 932:10-24.

24 Shimoda K, Sauve Y, Marini A, Schwartz JP, Commissiong JW: A high percentage yield of tyrosine hydroxylase-positive cells from rat E14 mesencephalic cell culture. Brain Res 1992;586:319-331.

25 Eriksson P: Developmental neurotoxicology in the neonate - Effects of pesticides and polychlorinated organic substances. Arch Toxicol Suppl 1996;18:81-88.
26 Eriksson P: Developmental neurotoxicity of environmental agents in the neonate. Neurotoxicology 1997;18:719-726.

27 Narahashi T: Nerve membrane ion channels as the target site of insecticides. Mini Rev Med Chem 2002;2:419-432.

28 Vijverberg HP, van der Zalm JM, van der BJ: Similar mode of action of pyrethroids and DDT on sodium channel gating in myelinated nerves. Nature 1982;295:601-603.

29 Lund AE, Narahashi T: Interaction of DDT with sodium channels in squid giant axon membranes. Neuroscience 1981;6:2253-2258.

30 Ferreira FM, Madeira VM, Moreno AJ: Interactions of 2,2-bis( $p$-chlorophenyl)-1,1-dichloroethylene with mitochondrial oxidative phosphorylation. Biochem Pharmacol 1997;53: 299-308.

31 Moreno AJ, Madeira VM: Mitochondrial bioenergetics as affected by DDT. Biochim Biophys Acta 1991;1060:166-174. 